\title{
Effects of Situational Demand Upon Social Enjoyment and Preference in Schizotypy
}

\author{
Stuart W. Quirk, Lakshmi Subramanian, and Michael Hoerger \\ Central Michigan University
}

\begin{abstract}
The socioemotional functioning of schizophrenic and schizotypic individuals is marked by withdrawal, poor organization, and limited emotional displays. Such behavioral tendencies and lack of social enjoyment in schizotypy could be linked to the relative situational demands or role ambiguity inherent in specific social activities. To determine whether high-schizotypy individuals prefer more clearly role-defined social activities (e.g., visiting relatives) to more ambiguous, novel situations (e.g., going alone to a party), the authors gathered reports from 52 high-schizotypy and 60 low-schizotypy individuals on their enjoyment and frequency of engaging in social situations varying in relative situational demand. Parallel reports were obtained from knowledgeable others. Group $\times$ Situational Demand interactions revealed the hypothesized pattern of reduced frequency and enjoyment ratings for ambiguous or novel situations by the high-schizotypy participants in both self and others' reports. Groups were more comparable in their reported frequency and enjoyment of less ambiguous situations. Results suggest the importance of situational demands in the socioemotional experience and behavioral withdrawal in schizotypy.
\end{abstract}

Keywords: schizotypy, social functioning, situational demand

Poor socioemotional functioning is a defining feature of schizophrenia and schizotypy. Individuals with schizophrenia and less severe spectrum psychopathology present with notable difficulties regulating emotions and managing social relationships. Observable difficulties include poor eye contact and social skills, oddness, hostility, and social withdrawal (Keltner \& Kring, 1998; Zborowski \& Garske, 1993). Despite the central role of social withdrawal and diminished emotional expressivity, individuals with schizophrenia and spectrum pathology report intact subjective responses to evocative stimuli, such as film clips, pictures, and odors (e.g., Horan, Green, Kring, \& Nuechterlein, 2006; Kring, Kerr, Smith, \& Neale, 1993; Quirk \& Strauss, 2001). It appears that the emotional dysfunction seen in these disorders is not well explained by deficits in basic emotional reactivity.

An individual's capacity to regulate emotions and orchestrate effective social behavior during complex and ambiguous situations requires substantial cognitive processing, including anticipation of others' behaviors, memory searches for behavioral repertoires, and the evaluation of the impact of various behaviors. As such, deficits in general and impaired social cognition processes are obvious candidates for understanding socioemotional deficits in schizophrenic spectrum psychopathology (Knight \& Valner, 1993; Penn, Combs, \& Mohamed, 2001). Indeed, the bulk of research regarding the causes of emotional dysfunction in schizophrenic spectrum

Stuart W. Quirk, Lakshmi Subramanian, and Michael Hoerger, Department of Psychology, Central Michigan University.

Lakshmi Subramanian is now at Department of Psychology, Larned State Hospital.

Correspondence concerning this article should be addressed to Stuart W. Quirk, Department of Psychology, 137 Sloan Hall, Central Michigan University, Mount Pleasant, MI 48859. E-mail: stuart.quirk@cmich.edu pathology has emphasized the importance of the cognitive processes involved in the complex evaluations, expectations, recollections, and motivational systems that underlie socioemotional behaviors (e.g., Gooding, Matts, \& Rollmann, 2006; Heinrichs, 2005). However, situational variables may also play an important role in the behaviors and experiences of individuals with schizophrenic spectrum pathology.

Levels of situational demand or role ambiguity are important determinants of social behavior and emotional experience (Shoda, Mischel, \& Wright, 1993). Situational demand refers to the extent that specific roles and competencies are required within a social setting (Bliese \& Castro, 2000). Clearly defined and predictable settings require behaviors that are relatively automatized and role specific (such as engaging in small talk while waiting for a bus or visiting with relatives). Role-ambiguous situations demand more novel and spontaneous behaviors (such as choosing how to best present oneself on a first date). People with schizophrenia and spectrum disorders may become overwhelmed in ambiguous situations as these place additional demands on cognitive faculties, such as those involved in self-regulation, anticipation, and metapsychological observation. Such situations are also more likely to evoke distress due to their unpredictability. Schizophrenia spectrum individuals may anticipate distress and poor performance in role-ambiguous social activities and thus avoid them.

Although this hypothesis has not been specifically evaluated with schizophrenia spectrum individuals, researchers have observed the negative effects of increased interpersonal stress on socioemotional functioning. The deleterious effects of interpersonally stressful interactions in schizotypy—such as being videotaped while being asked increasingly intrusive interview questions-are evidenced by behaviors that are less well organized, less facilita- 
tive, and more odd and hostile than comparison individuals (Shean \& Wais, 2000; Zborowski \& Garske, 1993).

Studies in the area of organizational and social psychology have established the relationship between role ambiguity and stress, and impaired performance (Fried, Ben-David, Tiegs, Avital, \& Yeverechyahu, 1998; Karasek, 1979). Karasek's (1979) demandcontrol model posited that role clarity ameliorates the demandstrain relationship. Situations in which demands are high but role clarity is also high are perceived as less negative and stressful than high-demand situations in which role clarity is low. These findings helped form the basis for the hypothesis that situations of greater role ambiguity will be described as less enjoyable by highschizotypy individuals who will, therefore, participate in such situations less frequently than comparison participants. As individuals high in schizotypic traits may be less able to complete social behavior and preference scales requiring metapsychological evaluation, the reports of friends and relatives were also obtained.

\section{Method}

\section{Participants}

Study participants were recruited from a large screening group of 1,152 students between the ages of 18 to 26 years who attended a large Michigan public university or a small liberal arts college in Wisconsin. Participants were given the Schizotypal Personality Questionnaire (SPQ; Raine, 1991), and those scoring in the top 10th percentile of the screening sample (scores over 36) were recruited to create a high-schizotypy group of 52; a random sample of 60 students scoring below the 50th percentile served as a comparison group. Participants with a history of epilepsy, organic brain damage, and/or substance abuse were excluded. Friends and relatives of participants were contacted via mail, e-mail, and phone. Data from a friend $(75 \%)$ or a relative $(25 \%)$ of the participants were obtained from 98 participants ( $88 \%$ of sample).

\section{Measures}

The SPQ is a 74-item true-false format questionnaire designed to assess the range of symptoms found in the Diagnostic and Statistical Manual of Mental Disorders (DSM-III-R; American Psychiatric Association, 1987) criteria for schizotypal personality disorder. The SPQ scores in the current sample had a full scale coefficient alpha of .96. The decision to define the high-schizotypy group as those who scored above the 90th percentile on the SPQ was based on Raine's (1991) finding that 55\% of those scoring in the top 10th percentile met $D S M-I I I-R$ diagnostic criteria for schizotypal personality disorder.

The Revised Social Anhedonia Scale (Eckblad, Chapman, Chapman, \& Mishlove, 1982) is a 40-item true-false self-report questionnaire designed to assess trait levels of decreased pleasure experienced from interpersonal sources. Scale items in the current sample demonstrated good internal consistency with a coefficient alpha of .89 .

Twenty-four items were selected from the social discomfort, extraversion, friendliness, and gregariousness scales from the International Personality Item Pool (Goldberg et al., 2006) to construct a trait social anxiety scale for the current study. Items included, "I seek solitude" and "I am comfortable in unfamiliar situations" (reverse coded). Participants rated each item on a 5-point Likert-type scale ranging from very inaccurate to very accurate. The scale scores yielded a coefficient alpha of .94.

The Social Activity Preference Scale was constructed for use in this study to assess participants' levels of enjoyment and frequency of engaging in 22 common social activities, such as "Talking to coworkers," "Watching TV with friends," and "Visiting relatives." Social activities varying in role ambiguity were rationally generated by the investigators with some items adapted from the Social Functioning Scale (Birchwood, Smith, Cochrane, Wetton, \& Copestake, 1990). Twenty judges provided ratings for these items in terms of how much each social activity "involves predictable, familiar, structured interactions versus unfamiliar or unpredictable situations which may require novel responses" using a 7-point Likert-type scale ranging from low predictability/structure to high predictability/structure. Judges included 14 doctoral-level psychologists (the majority of whom have published in the area of personality psychology), three graduate students in clinical psychology, and three undergraduate assistants working in Stuart W. Quirk's research lab. Experts were reliable in their situational demand ratings (intraclass correlation coefficient $=.88$ ). Summed scale expert ratings by doctoral-level psychologists were comparable to those made by student raters, $t(18)=0.68, n s$. Situations rated as more predictable and role defined included "Going to a movie with friends," "Making dinner for a friend," and "Attending a formal occasion." More role ambiguous and novel situations included "Going to a bar alone," "Joining a new social group," and "Going to a party alone."

Study participants were asked to rate how much they enjoy each activity on a 5-point Likert-type scale ranging from do not enjoy the activity at all to greatly enjoy the activity. Participants also indicated how many times they had engaged in each of the activities in the prior 30 days. Friends and relatives of the participants were asked to complete a parallel version of this scale. The scale demonstrated high internal consistency for self-reported enjoyment ( $\alpha=.84)$ and frequency $(\alpha=.86)$, and for others' reports of enjoyment $(\alpha=.97)$ and behavioral frequency $(\alpha=.81)$. Selfreport ratings were highly correlated with others' reports for enjoyment $(r=.79)$ and for frequency $(r=.77)$. An additional group of 26 unselected undergraduates provided test-retest data with a 3to 4 -week interval. Time 1 and Time 2 enjoyment ratings were highly correlated $(r=.82)$, as were frequency reports $(r=.95)$.

We administered a demographic and family history form to the participants to determine whether first-degree relatives experienced signs and symptoms suggestive of psychopathology. Participants indicated whether anyone in their immediate biological family suffered from any of the following symptoms, grouped into sets of three items:

1. anxiety: intense distress, phobic fears, constant worry over minor things;

2. depression: prolonged deep sadness, low energy, suicide attempt or suicidal thoughts;

3. psychotic symptoms: disorganized speech, hearing voices, strongly held false beliefs; or

4. problem drinking: excessive drinking leading to job loss, 
family problems from drinking, and daily drinking for months at a time.

Two participants failed to complete this instrument.

\section{Procedures}

Participants provided written consent separately for the screening procedure and before completing the laboratory portion of the study. Screenings were conducted in introductory level courses in psychology and other departments, including history, English, and art. Those recruited for the second phase completed a questionnaire packet. Afterwards, each participant was asked to provide names and contact information of three close friends and/or relatives. Participants were informed that the friends or relatives would be contacted in order to provide information regarding the participant's social preferences, social behavior, and perceived enjoyment of social activities. None of the participants objected to providing this information.

Friends and relatives were mailed copies of the Social Activity Preference Scale along with a cover letter. Those who did not respond by mail were contacted by phone and e-mail. Of the 98 collateral reports, 34 were obtained by mail, 46 by phone, and 18 via e-mail. Those contacted via phone were requested to write down the scale values and labels to aid in completing the items.

\section{Data Analyses}

Two approaches were used to analyze the data. The first approach involved the construction of a data set in which the 22 Social Activity Preference Scale situations were treated as items. Mean expert ratings of role ambiguity were entered for each item. Mean enjoyment and frequency reports were computed for each item separately for the high-schizotypy and comparison groups (for both self and others' reports). Support for the hypothesized pattern would be revealed in strong, positive correlations between expert ratings and enjoyment and frequency reports for the highschizotypy group members reflecting greater preference for and engagement in low-ambiguity situations.

For the second approach to the data analysis, expert ratings were used to identify high, medium, and low role-ambiguous situations. The seven highest and lowest rated items were used to create high and low role-ambiguity scores, and the remaining eight items served as medium-level items. Scores from these three levels were then analyzed with a repeated-measures multivariate analysis of variance (MANOVA). Social anxiety and social anhedonia scores were used as covariates to determine whether these socially relevant individual differences would account for the obtained results. The hypothesized relationship between level of role ambiguity and group membership should be revealed in Group $\times$ Level of Ambiguity interactions. High-schizotypy and comparison participants should be similar in their reported enjoyment and participation in low-ambiguity situations, but high-schizotypy group members should report less enjoyment and engagement in highly ambiguous situations.

\section{Results}

The groups consisted of primarily White participants (75\%). The groups were similar in age $(M=20.2, S D=2.3), t(110)=$ $-1.68, p=.10$, and had comparable numbers of women $(53 \%$ in both groups). Consistent with the expectation that schizotypy exists on the vulnerability continuum with schizophrenic pathology, high SPQ scoring individuals reported a greater number of psychotic symptoms in their first-degree relatives $(M=0.35$, $S D=0.76)$ than comparison group members $(M=0.09, S D=$ $0.34), t(108)=-2.35, p<.02, d=0.45$. Groups reported comparable levels of anxiety and depression in their immediate family (larger $d=0.20$ ). High-schizotypy participants reported somewhat greater numbers of substance abuse symptoms in family members, $t(108)=-1.51, p<.14, d=0.29$.

Collateral contact information was gathered from more friends than family for both the high-schizotypy group (73\%) and comparison participants $(76 \%)$. Friends were more accessible than parents or relatives who either were less willing to provide information or could not be contacted. Of those contacted, $47 \%$ knew the participants for at least 6 months, $17 \%$ for at least 2 years, and $21 \%$ knew the participants for their lifetime. Years of acquaintance did not differ by group status, $\chi^{2}(2, N=98)=0.75, p=.69$, and the format of data collection (phone, mail, e-mail) was unrelated to others' ratings, larger $F(2,98)=0.14$, ns.

The first data analytic method involved treating scale items as cases in a data set that included the mean expert rating for each item. Mean self and others' reports for each item were computed separately for high-schizotypy and comparison group members. Strong support was obtained for the hypothesis that high-schizotypy participants would enjoy and engage in role-defined activities more than ambiguous activities as expert ratings were strongly correlated with highschizotypy group members' reported enjoyment $(r=.68, p<.01)$ and frequency $(r=.53, p<.01)$. Similarly strong correlations were observed between expert ratings and others' reports of enjoyment $(r=.72, p<.01)$ and frequency $(r=.54, p<.01)$ for the high-schizotypy individuals. In contrast, none of the self or others' reports were reliably related to expert ratings for the comparison group (average $r=.09$, largest $r=.29, p=.20$ ). $r$ to $Z$ transformations were used to determine that all of the between group independent correlations reliably differed at the $p<.01$ level.

Strong support for the primary hypothesis was also obtained when reported enjoyment and frequency ratings were compiled into high, medium, and low role-ambiguous situation scales as determined by expert ratings. As the assumption of equality of variances across levels of the repeated measures was not met for a number of the analyses, the more conservative MANOVA model was used in the subsequent analyses as this model treats levels as independent variables and is more robust against violations of sphericity.

A large interaction effect accounting for $34 \%$ of the variance was observed between groups across levels of role ambiguity for self-reported enjoyment, $F(2,109)=27.87, p<.001, \eta^{2}=.34$. A main effect was observed for level of ambiguity, $F(2,109)=$ 38.08, $p<.001, \eta^{2}=.41$, as participants reported less enjoyment in highly ambiguous activities $(M=3.30, S D=1.13)$ than in medium-ambiguity situations $(M=3.84, S D=0.74), t(111)=$ $-7.63, p<.001$. Medium- and low-ambiguity situations $(M=$ $3.74, S D=0.58$ ) were similar in rated enjoyment, $t(111)=-1.93$, $p<.07$. High-schizotypy participants reported less social enjoyment than comparison individuals, $F(1,110)=44.46, p<.001$, $\eta^{2}=.29$. Group $\times$ Ambiguity Level data for self-reported enjoyment and frequency are presented in Figure 1. The groups displayed the expected pattern of larger differences at higher levels of 
Self Reported Enjoyment
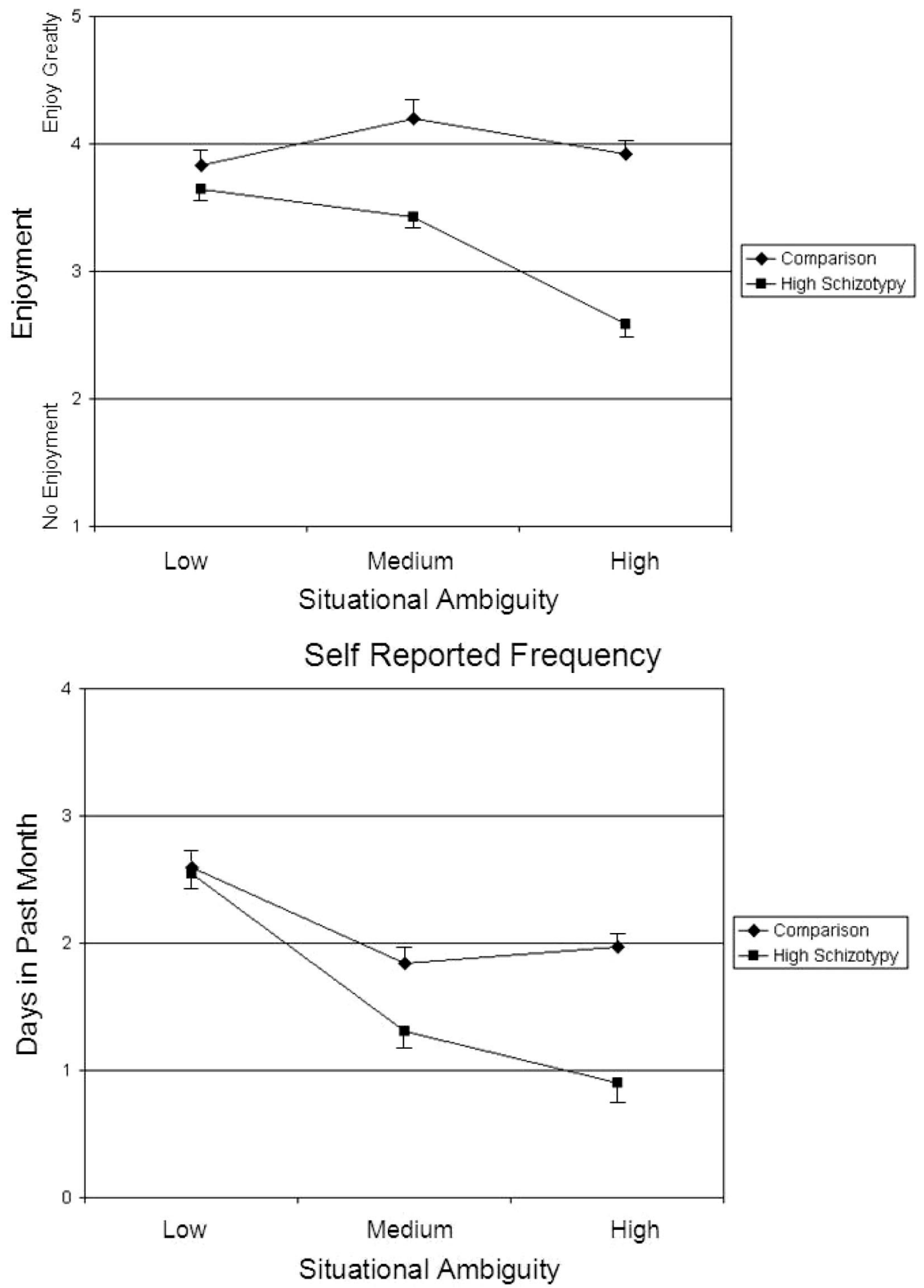

Figure 1. Mean $( \pm S E)$ self-reported enjoyment and frequency of engagement in social activities (in past 30 days) of varying situational ambiguity by high-schizotypy and comparison group participants. 
role ambiguity. High-schizotypy group members were somewhat lower in their enjoyment of low-ambiguity situations $(d=0.33)$ but demonstrated large effect size differences in enjoyment of medium-ambiguity situations $(d=1.20)$ and high-ambiguity situations $(d=1.45)$.

A large interaction effect was also observed between groups across levels of ambiguity for self-reported frequency of engaging in the situations during the past 30 days, $F(2,109)=19.02, p<$ $.001, \eta^{2}=.26$. Participants reported decreasing frequency of engaging in situations of higher ambiguity, $F(2,109)=99.54, p<$ $.001, \eta^{2}=.65$. Participants did not differ in their frequency of engaging in low- and medium-ambiguity activities $(M=1.47$, $S D=1.05 ; M=1.59, S D=0.71$, respectively), $t(118)=-1.59$, $p>$.11. Participants reported engaging in the greatest number of low-ambiguity activities $(M=2.56, S D=1.00), t(118)=11.64$, $p<.001$ (compared to medium-ambiguity situations). Highschizotypy participants reported less frequent social participation than comparison individuals, $F(1,110)=15.78, p<.001, \eta^{2}=$ .13. Groups displayed the expected pattern of increasing differences at higher levels of situational ambiguity. Groups were comparable in their reported frequency of engaging in low-ambiguity situations $(d=0.04)$ with a large effect size discrepancy for medium-ambiguity situations $(d=0.73)$, and the largest difference observed between groups for highly role-ambiguous situations $(d=1.18)$.

Others' reports regarding perceived enjoyment and behavior of participants yielded results parallel to self reports. The expected interaction effect was observed between groups across levels of situational demand for reported enjoyment, $F(2,94)=32.80, p<$ $.001, \eta^{2}=41$. Main effects were observed for situational ambiguity, $F(2,94)=43.61, p<.001, \eta^{2}=.48$, and group membership, $F(1,95)=69.94, p<.001, \eta^{2}=.42$. Group $\times$ Ambiguity Level data for others' perceived enjoyment and behavioral frequency are presented in Figure 2. Reports conformed to the overall hypothesized pattern; however, high-schizotypy participants were seen as enjoying low-ambiguity situations less than comparison group members $(d=0.63)$. The differences between groups at higher ambiguity levels were more extreme as the high-schizotypy group was described as enjoying medium-demand situations substantially less than comparison participants $(d=1.38)$, with the largest effect size difference of nearly two standardized mean differences observed between the groups' enjoyment ratings of high-ambiguity situations $(d=1.93)$.

The expected interaction effect was also observed for others' behavioral frequency reports, $F(2,95)=8.82, p<.001, \eta^{2}=.16$. Main effects were observed for ambiguity, $F(2,95)=97.78, p<$ $.001, \eta^{2}=.67$, and group membership, $F(1,96)=27.49, p<$ $.001, \eta^{2}=.22$. Differences in frequency of low-ambiguity situations by group membership displayed a modest effect $(d=0.38)$. High-schizotypy participants were described as engaging in medium-ambiguity situations less often than comparison participants $(d=0.88)$ with the largest effect size difference observed between groups for the highly ambiguous situations $(d=1.42)$.

Additional analyses were conducted to determine whether the obtained pattern of results could be attributed to the responses of those reporting the fewest symptoms on the SPQ, accounted for by trait levels of social anxiety or social anhedonia, or if they were associated with specific schizotypy symptom dimensions of the SPQ. The lowest scoring SPQ participants (those scoring greater than one $S D$ below the sample mean, SPQ scores $<9, n=38$ ) were compared to those reporting SPQ symptoms within one $S D$ of the sample mean $(n=23)$ to determine whether the pattern of results was accounted for by those reporting very few symptoms. These two groups were comparable in self and others' reports with no significant interactions (mean $\eta^{2}=.05$, largest $\eta^{2}=.08$ ) or main effects (mean $\eta^{2}=.004$, largest $\eta^{2}=.006$ ).

Greater trait social anxiety was associated with reports of less activity enjoyment for self $(r=-.43)$ and others $(r=-.44)$. Social anxiety was also related to lower levels of reported behavioral frequency for self $(r=-.29)$ and others $(r=-.37)$. Greater social anhedonia was associated with less self-reported enjoyment $(r=-.33)$ and others' reported enjoyment $(r=-.35)$ but was not reliably related to frequency reports for self $(r=-.02)$ and others $(r=-.14)$. To determine whether these individual difference variables could account for the Group $\times$ Role Ambiguity interaction effects, they were each entered as covariates in a series of repeated measures MANOVA's with group membership and the three levels of role ambiguity scores. Social anxiety scores did not generate any significant interaction effects for either self or others' reports of either enjoyment or behavioral frequency (largest $\eta^{2}=$ $.02)$. Social anhedonia was a significant covariate in one analysis and generated a modest anhedonia by role ambiguity interaction effect for others' reports of enjoyment $\left(\eta^{2}=.05\right)$, but the highschizotypy Group $\times$ Role Ambiguity interaction in that analysis remained significant and substantial $\left(\eta^{2}=.27\right)$.

We created high and low groups for each of the three symptom dimensions of the SPQ (cognitive-perceptual, interpersonal, and disorganization) using median splits. The hypothesized interaction effect of less enjoyment and lower frequency of more roleambiguous situations by high-symptom groups was found across symptom dimensions with large effect sizes observed for both self and others' reports. For example, the interaction effect size observed for cognitive-perceptual symptoms by role ambiguity for self-reported enjoyment was large $\left(\eta^{2}=.27\right)$. The parallel interaction effect sizes were $\eta^{2}=.29$ for interpersonal symptoms and $\eta^{2}=.21$ for disorganization symptoms. Effect sizes for self-report data ranged from $\eta^{2}=.10$ to $\eta^{2}=.29$, and from $\eta^{2}=.07$ to $\eta^{2}=$ .33 for others' reports.

\section{Discussion}

The current study was designed to assess the influence of situational demand and role ambiguity in the experienced pleasure and social behaviors of individuals high in schizotypy. The overall hypothesis that situations judged to involve role ambiguity and novelty would be experienced as less enjoyable and engaged in less frequently by high-schizotypy participants received strong support in both self reports and in data obtained from knowledgeable others. The pattern was observed across symptom dimensions and was not accounted for by social anhedonia or social anxiety reports.

The results of the current study suggest a nuanced view of social withdrawal in schizotypy compared to the global social withdrawal and anhedonia often assessed via self report and codified in diagnostic criteria. Instead, high-schizotypy participants in the current study reported specificity in their interest and enjoyment of social situations. This preference may be linked to situation-specific motivations, personality, and/or informational processing deficits 
Others' Reported Enjoyment
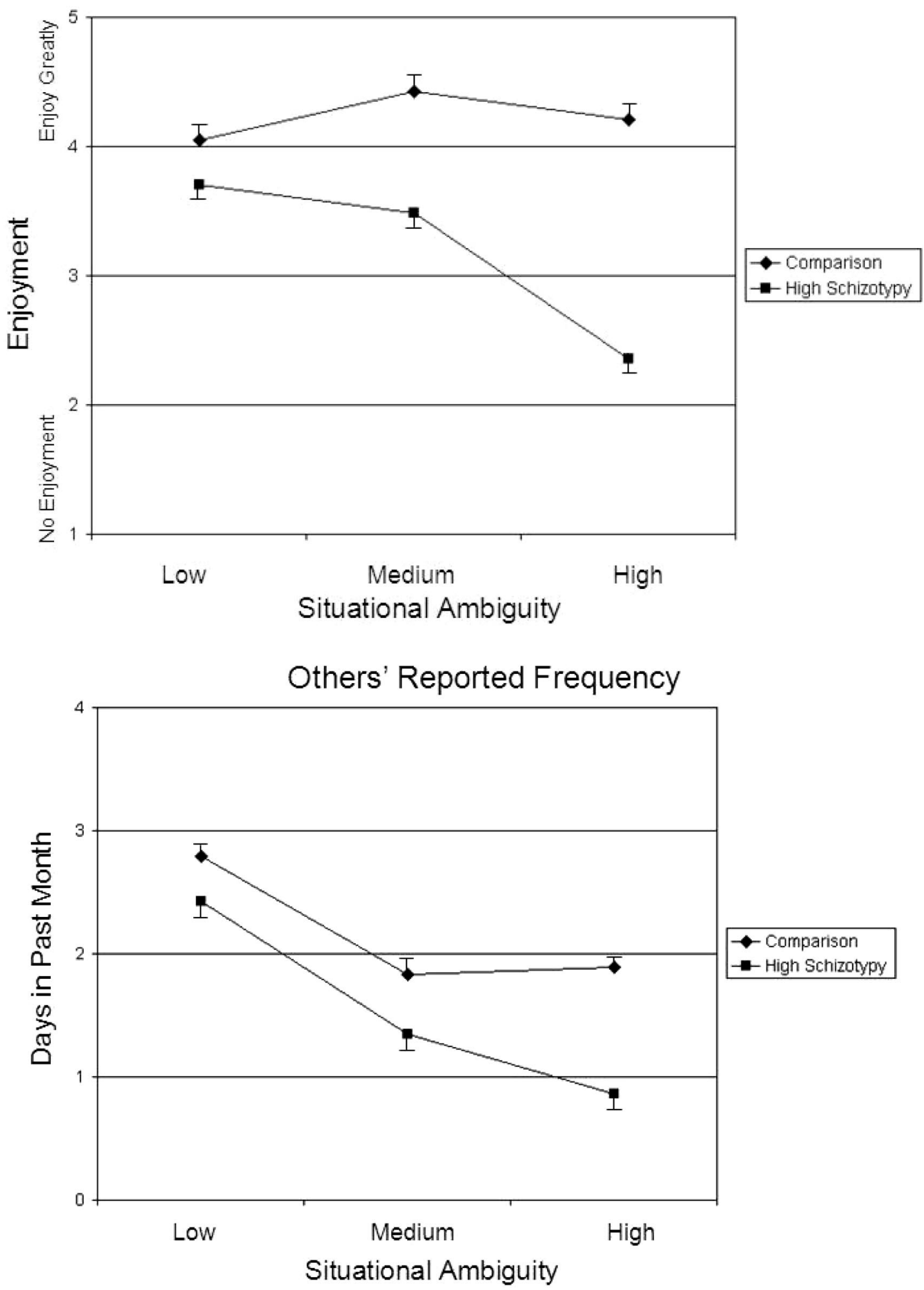

Figure 2. Mean $( \pm S E)$ others' reported enjoyment and frequency of engagement in social activities of varying situational ambiguity for high-schizotypy and comparison group participants. 
observed in schizotypy. A number of researchers and theoreticians have focused on such processes that may help explain the notable socioemotional deficits in schizophrenia and spectrum psychopathology. Candidate processes that have received attention include personality traits, such as trait sensitivity to emotional stimulation, and processing deficits, including cognitive and social recognition deficits (e.g., Gooding et al., 2006; Horan et al., 2006; Kerns, 2005; Penn et al., 2001).

Individuals high in schizotypy have cognitive deficits similar in form but of less severity to those found in schizophrenia (Lenzenweger, 1998). Identified cognitive deficits, including impaired working memory, attentional dysfunction (e.g., Barch et al., 2004), and deficits in goal recognition (Leonhard \& Corrigan, 2001), may contribute to a preference for social situations that place fewer demands on information processing capacities. When in ambiguous interpersonal situations, poor attention, memory, and cognitive organizational skills may impair schizotypic individuals' performance and, consequently, lead to unsuccessful and unsatisfying experiences. These experiences may contribute to patterns of social withdrawal and isolation. However, highly structured situations provide clear expectations that evoke less anxiety and increase chances of successful performance. Examining the relationships between deficits in social cognition, memory, and attention with situational demand characteristics may help illuminate the interface between intra- and interindividual variables of emotional regulation in schizotypy.

The results from the current study indicate substantial differences in emotional reports of pleasure between high-schizotypy and comparison individuals; however, various previous studies have found few abnormalities in the emotional reactivity of schizophrenic and schizotypic individuals. The discrepancy in results from laboratory settings may be explained, in part, by the amount of situational demand provided by the investigators. A number of previous studies designed to measure emotional reactivity have relied upon Likert-type scales to measure emotional responses to evocative stimuli, such as film clips and odors (e.g., Berenbaum \& Oltmanns, 1992; Horan et al., 2006), or sets of evocative pictures (e.g., Quirk, Strauss, \& Sloan, 1998). The high demand and structure in these research designs may limit the measurable disturbances in emotional reactivity of schizophrenia patients and spectrum individuals.

Results from this study relied on paper-pencil measures. This study also relied on retrospective self reports, which are vulnerable to impression management and memory biases. This concern is somewhat mitigated as others' reports should be less prone to these biases. Interpretation of the results in the current study is limited by the possible role of unmeasured symptom variables such as depressive symptoms. For example, the poor concentration and fatigue that characterize depression may help account for diminished interest and engagement in novel social activities that involve greater effort and cognitive challenges than do clearly roledefined activities. The finding that measures of social anxiety and social anhedonia did not account for the results helps to ameliorate this problem somewhat. Also, affiliating with strangers versus family or friends appears to covary with ambiguity ratings. Assessing role demand through laboratory manipulation would provide a stronger test of the hypothesis and conclusions presented here.

\section{References}

American Psychiatric Association. (1987). Diagnostic and statistical manual of mental disorders (3rd ed., rev.). Washington, DC: Author.

Barch, D. M., Mitropoulou, V., Harvey, P. D., New, A. S., Silverman, J. M., \& Siever, L. J. (2004). Context-processing deficits in schizotypal personality disorder. Journal of Abnormal Psychology, 113, 556-568.

Berenbaum, H., \& Oltmanns, T. (1992). Emotional experience and expression in schizophrenia and depression. Journal of Abnormal Psychology, 101, 37-44.

Birchwood, M., Smith, J., Cochrane, R., Wetton, S., \& Copestake, S. (1990). The social functioning scale: The development and validation of a new scale of social adjustment for use in family intervention programmes with schizophrenia. British Journal of Psychiatry, 157, 853859.

Bliese, P. D., \& Castro, C. A. (2000). Role clarity, work overload, and organizational support: Multilevel evidence of the importance of support. Work and Stress, 14, 65-73.

Eckblad, M. L., Chapman, L. J., Chapman, J. P., \& Mishlove, M. (1982). The revised social anhedonia scale. Unpublished test, University of Wisconsin, Madison.

Fried, Y., Ben-David, H. A., Tiegs, R. B., Avital, N., \& Yeverechyahu, U. (1998). The interactive effect of role conflict and role ambiguity on job performance. Journal of Occupational and Organizational Psychology, $71,19-27$.

Goldberg, L. R., Johnson, J. A., Eber, H. W., Hogan, R., Ashton, M. C., Cloninger, C. R., \& Gough, H. C. (2006). The international personality item pool and the future of public-domain personality measures. Journal of Research in Personality, 40, 84-96.

Gooding, D. C., Matts, C. W., \& Rollmann, E. A. (2006). Sustained attention deficits in relation to psychometrically identified schizotypy: Evaluating a potential endophenotypic marker. Schizophrenia Research, $82,27-37$.

Heinrichs, R. (2005). The primacy of cognition in schizophrenia. American Psychologist, 60, 229-242.

Horan, W., Green, M., Kring, A., \& Nuechterlein, K. (2006). Does anhedonia in schizophrenia reflect faulty memory for subjectively experienced emotions? Journal of Abnormal Psychology, 115, 496-508.

Karasek, R. A. (1979). Job demands, job decision latitude, and mental strain: Implications for job redesign. Administration Science Quarterly, 24, 285-307.

Keltner, D., \& Kring, A. M. (1998). Emotion, social function, and psychopathology. Review of General Psychology, 2, 320-342.

Kerns, J. (2005). Positive schizotypy and emotional processing. Journal of Abnormal Psychology, 114, 392-401.

Knight, R. A., \& Valner, J. B. (1993). Affective deficits in schizophrenia. In C. G. Costello (Ed.), Symptoms of schizophrenia (pp. 145-200). New York: Wiley.

Kring, A. M., Kerr, S. L., Smith, D. A., \& Neale, J. M. (1993). Flat affect in schizophrenia does not reflect diminished subjective experience of emotion. Journal of Abnormal Psychology, 102, 507-517.

Lenzenweger, M. (1998). Schizotypy and schizotypic psychopathology: Mapping an alternative expression of schizophrenia liability. In M. Lenzenweger \& R. Dworkin (Eds.), Origins and development of schizophrenia: Advances in experimental psychopathology (pp. 93-122). Washington, DC: American Psychological Association.

Leonhard, C., \& Corrigan, P. W. (2001). Social perception in schizophrenia. In P. W. Corrigan \& D. L. Penn (Eds.), Social cognition and schizophrenia (pp. 73-95). Washington, DC: American Psychological Association.

Penn, D. L., Combs, D. R., \& Mohamed, S. (2001). Social cognition and social functioning in schizophrenia. In P. W. Corrigan \& D. L. Penn (Eds.), Social cognition and schizophrenia (pp. 97-121). Washington, DC: American Psychological Association.

Quirk, S., \& Strauss, M. (2001). Visual exploration of emotion eliciting 
images by patients with schizophrenia. Journal of Nervous and Mental Disease, 189, 757-765.

Quirk, S., Strauss, M., \& Sloan, D. (1998). Emotional response as a function of symptoms in schizophrenia. Schizophrenia Research, 32, 31-39.

Raine, A. (1991). The Schizotypal Personality Questionnaire (SPQ): A measure of schizotypal personality based on DSM-III-R criteria. Schizophrenia Bulletin, 17, 555-564.

Shean, G., \& Wais, A. (2000). Interpersonal behavior and schizotypy. Journal of Nervous and Mental Disease, 188, 842-846.

Shoda, Y., Mischel, W., \& Wright, J. C. (1993). The role of situational demands and cognitive competencies in behavior organization and personality coherence. Journal of Personality and Social Psychology, 65, 1023-1035.

Zborowski, M. J., \& Garske, J. P. (1993). Interpersonal deviance and consequent social impact in hypothetically schizophrenia-prone males. Journal of Abnormal Psychology, 102, 482-489.

Received September 27, 2006 Revision received February 22, 2007

Accepted February 23, 2007

\section{E-Mail Notification of Your Latest Issue Online!}

Would you like to know when the next issue of your favorite APA journal will be available online? This service is now available to you. Sign up at http://watson.apa.org/ notify/ and you will be notified by e-mail when issues of interest to you become available! 
